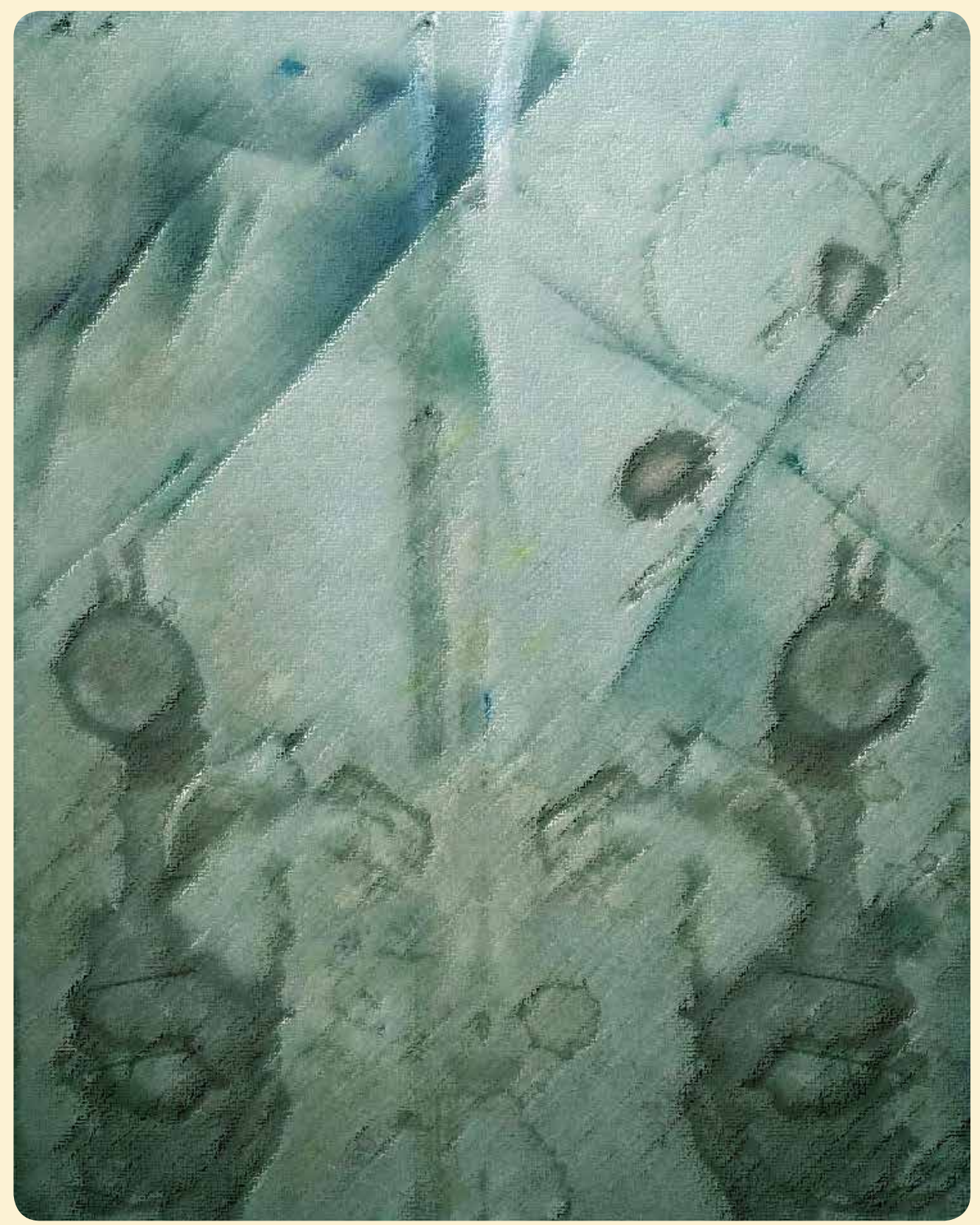

Apariciones Jamquy. Fotografía: Jorge Javier Muñoz (2019). 


\title{
Cuerpos envejecidos en corporalidades rarámuri. Entre la cotidianidad y la exclusión social de un grupo étnico del norte de México'
}

Aged bodies in rarámuri corporalities. Between everyday life and the social exclusion of an ethnic group from northern México // Corpos idosos em corpos rarámuri. Entre a vida cotidiana e a exclusão social de um grupo étnico do norte do México

\author{
Laura Vázquez Vega ${ }^{2}$ \\ Escuela Nacional de Antropología e Historia, ENAH, México \\ laurayvazvega@gmail.com
}

Revista Corpo-grafías: Estudios críticos de y desde los cuerpos / Volumen 7 - Número 7 / Enero - diciembre 2020 / ISSN impreso 2390-0288, ISSN digital 2590-9398 / Bogotá, D.C., Colombia / 117-129

\section{(9) $(1) \Theta$}

Fecha de recepción: 4 de marzo de 2019

Fecha de aceptación: 21 de junio de 2019

DOI: https://doi.org/10.14483/25909398.15510

Cómo citar este artículo: Vázquez Vega, L. (2020, enero-diciembre). Cuerpos envejecidos en corporalidades rarámuri. Entre la cotidianidad y la exclusión social de un grupo étnico del norte de México. Revista Corpo-grafías: Estudios críticos de y desde los cuerpos, 7(7), pp. 117-129 / ISSN 2390-0288.

\footnotetext{
Articulo de Investigación: El presente artículo es un avance de la investigación doctoral 2018-2021, que llevo a cabo en la línea Antropología en salud, Ciencias sociomédicas, adscrita a la Facultad de Medicina, Universidad Nacional Autónoma de México.

Doctoranda Antropología en salud, Facultad de Medicina - UNAM, maestra Antropología Física y licenciada Etnología, ENAH. Coordinadora académica Licenciatura en Etnología, ENAH. Directora Museo Culturas de Norte y Zona Arqueológica Paquimé, Chihuahua, INAH. Docente nivel licenciatura en temas sobre métodos y técnicas etnográficas, atlas etnográfico y teorías antropológicas desde el 2004.
} 


\section{Resumen}

Avance de investigación inicial sobre los saberes, creencias, recodificaciones y resignificaciones de cómo se percibe, vive y transita hacia el envejecimiento rarámuri en términos de salud, enfermedad y atención, considerando las desventajas acumuladas debajo de la piel, desde un enfoque intercultural que considere las narrativas, actitudes, prácticas y comportamientos entre géneros y generaciones en Munérachi, municipio de Batopilas, Chihuahua.

\section{Palabras clave}

Envejecimiento; tarahumaras; curso de vida; desventajas acumuladas

\section{Abstract}

Advance of initial investigation on the knowledge, belie$\mathrm{fs}$, recodifications and resignifications of how it is perceived, lived and transited the rarámuri aging in terms of health, illness and attention, considering the disadvantages accumulated under the skin, from an intercultural approach that considers the narratives, attitudes, practices and behaviors between genders and generations in Munérachi, municipality of Batopilas, Chihuahua.

\section{Keywords}

Aging; Tarahumara; life course; accumulated disadvantages

\section{Resumo}

Avanço da investigação inicial sobre os conhecimentos, crenças, recodificações e ressignificações de como se percebe, vive e transita para o envelhecimento rarámuri em termos de saúde, doença e atenção, considerando as desvantagens acumuladas a partir de uma abordagem intercultural que considera a narrativas, atitudes, práticas e comportamentos entre gêneros e gerações em Munérachi, município de Batopilas, Chihuahua.

\section{Palavras-chave}

Envelhecimento; Tarahumara; curso de vida; desvantagens acumuladas

\section{Introducción}

El envejecimiento y la vejez son fenómenos generalizados, tendencias que no han encontrado aún la diversidad de respuestas suficientes, y que a su vez integren los múltiples referentes culturales y sociales para vivir esta etapa del ciclo de vida con salud, bienestar y calidad de vida, tanto en el ámbito de lo global como de lo local. Considerando, además, una expectativa de vida libre de cualquier restricción que limite las actividades cotidianas y una esperanza de vida con menos restricciones atribuibles a deficiencias funcionales físicas o mentales en este grupo de población en aumento.

En relación con el proceso de envejecimiento estandarizado, cabe señalar que la población del sector de más de 60 años se ha duplicado desde 1980, por lo que se prevé que el número de personas en todo el planeta sea alrededor de 2000 millones de personas para el 2050 (OMS, 2015). Estas cifras pueden considerarse como un triunfo del desarrollo, ya que las personas pueden vivir por más tiempo. No obstante, la mejora de la nutrición, el saneamiento, los avances médicos, la atención de la salud, la educación y la supuesta economía de bienestar, entre otros, no son generalizados en las poblaciones ni en todas las culturas. Incluso no se considera la edad en la vejez como un referente culturalmente definible, cuando en algunos grupos indígenas esta inicia alrededor de los 40 años y en donde la expectativa de vida no va más allá de los 60 años.

Elementos que sin duda restringen posibles beneficios tanto en lo individual como en lo colectivo, generando así incógnitas sobre la sobrevivencia de estos pese a 
las desventajas acumuladas incrustadas bajo la piel y el proceso multidimensional de la exclusión social, a saber, qué le ocurre al cuerpo conforme este envejece, por qué el deterioro es evidente en el funcionamiento corporal de unos y no tan obvio en otros, cómo ciertas fuerzas sociales pueden incidir en lo psicológico y biológico de los cuerpos, por qué la inequidad conduce a marcadores del envejecimiento y puede ser predictivo de la senescencia, por qué la estructura social vulnerabiliza y compromete la supervivencia de generaciones futuras y prepara las exclusiones del mañana, entre otras.

De tal manera, el hecho de que la población progresivamente se esté haciendo vieja genera, en la que suscribe, una preocupación académica sobre la representación del envejecimiento rarámuri y referentes asociados a su salud, enfermedad y formas de atención desde los saberes, creencias, recodificaciones y resignificaciones, así como las manifestaciones y formas de exclusión con la consecuente vulnerabilización como etnia y como grupo etario en la ranchería de Munérachi, Batopilas, Chihuahua.

\section{Planteamiento del problema y objetivos}

La forma en que las personas entienden, viven y habitan su cuerpo responde a una determinada construcción cultural del mismo, siendo fundamental la etapa del ciclo de vida en el que se encuentren, la relación actor-contexto y por consiguiente las estructuras sociales, políticas y económicas que la contengan. Los sistemas de creencias son reflejo de la visión del mundo que determinado colectivo tiene con respecto a su entorno y al papel del ser humano dentro de él, en donde la búsqueda del bienestar es el leitmotiv que moviliza. Esto es, se hace referencia a un entendimiento cultural y social, donde individualmente se define lo que significa estar-bien. La idea de 'bien estar' es una concepción que puede tomar distintas formas, una de ellas es el bienestar físico o de la salud que, además, articula un aparato simbólico que alimenta las ideas de lo sano y lo enfermo, lo que se ve envuelto en dinámicas de continuidades, rupturas y reinvenciones; base para que hombres y mujeres tomen posesión de su mundo. Lluís Duch (2002), antropólogo español, sugiere que:

Salud y enfermedad, además de ser expresiones de la situación del individuo en su mundo cotidiano, ponen de manifiesto el estado de aquellas relaciones humanas que permiten la constitución del tejido social, el cual es el ámbito en cuyo interior se despliega la convivencia como haz de tensiones, deseos, solidaridades, competencias y afirmaciones [...] la enfermedad y la sanación pueden considerarse como dos formas radicalmente distintas del clima social y emocional, con retóricas propias, que inciden decisivamente sobre los comportamientos de los individuos y los grupos humanos. (p.31)

Si a este imaginario le sumamos un par de factores como la cualidad étnica, el ser rarámuri, y el ciclo vital, concretamente el envejecimiento, ser viejo (a), ochérame o wiráami, respectivamente, nos encontramos con otras especificidades que impactan no solo la respuesta fisiológica de los cuerpos sino la manera en que ello se significa individual y colectivamente, lo que evidentemente implica la comprensión y valoración de: a) dicha cultura local con sus vínculos interculturales entre warijó, guarijíos, ódame, tepehuanes, o'oba, pimas bajos y mestizos, a partir del empleo de la lengua tradicional rarámuri, así como los recursos humanos, materiales y simbólicos asociados a su medicina y, por supuesto, b) la relación estructural que los 'inferioriza', separa y excluye como etnia, generación y género en las diferentes aristas de su cotidianidad con respecto a los centros de poder, los recursos y los valores dominantes. De tal manera, dichas experiencias tendrán una significación a partir del lenguaje mismo, mediante el sistema discursivo que les da sentido, siendo así los relatos, entramados de significado en estructuras sociales y condiciones materiales concretas que se simbolizan (Geertz, 2006). 
Ante este somero panorama se lleva a cabo una investigación interdisciplinaria que contempla la interacción y conexión entre saberes, creencias, recodificaciones y resignificaciones, de cómo se percibe y vive el envejecimiento, la salud, la enfermedad y la manera de atención entre hombres y mujeres rarámuri en las localidades referidas. Cabe señalar, que se desarrollará un análisis crítico de las narrativas sobre la vejez para comprender, por un lado, los múltiples referentes culturales, científicos y disciplinares al interior de un contexto histórico específico y, por el otro, las diversas lecturas del pasado y las recientes, mismas que inciden en la manera en que cada persona se advierte y se percibe; todo ello en un plano comparativo para destacar los significados que atribuyen en cada crónica. Así pues, las representaciones y los contextos discursivos se imprimen en la subjetividad de dichos relatos.

Como parte del proceso para problematizar el tema de interés, se reconstruirá conceptualmente a los sujetos de participación de este estudio para después contrastarlos con el dato empírico y redimensionarlos en su interpretación será fundamental aludir a interrelaciones en lo individual, familiar, comunitario, social, institucional (local y regional), geográfico, histórico, político y económico. A partir de ello es que se articularon las siguientes preguntas de investigación, a saber: a) Cuál es el proceso y las configuraciones sobre el envejecimiento rarámuri, con clave de género y de manera intergeneracional, ante la mirada rarámuri y fuera de dicha matriz cultural y b) Cuál es el punto de inflexión o transición asociado al cuerpo, la salud y el envejecimiento, así como la trayectoria del viejo(a) rarámuri "sano" y "enfermo" desde la epidemiología biomédica y sociocultural.

Considero que el tema que nos ocupa tiene relevancia y pertinencia académica y social, ya que el énfasis está en la salud desde la interculturalidad, pues comprende a uno de los grupos que se vulnera y excluye principalmente en dos sentidos: el primero por su particularidad lingüística y étnica y el segundo por su cualidad etaria, sin obviar el sentido de la violencia estructural, en lo general. Lo que sin duda puede contribuir a la invaluable trayectoria de los estudios sobre los determinantes sociales del proceso salud/enfermedad/atención (s/e/a), bajo un modelo de análisis que correlacione las causas, vínculos, distribución, regularidad y control de los múltiples factores que se asocian con la salud, a través del curso de vida, además de lo referente a las desigualdades estructurales como la extrema pobreza, la marginación y la distribución de la riqueza, por señalar algunas. Con la finalidad de evidenciar las causas biológicas, psicológicas y sociales que deben convenirse en la salud y las políticas públicas para acercarse oportunamente a propuestas que deriven en niveles de intervención para el bien estar y la calidad de vida en el ámbito de lo local, en términos de acción y práctica efectiva que no vulnere más ni la salud ni la integridad de este pueblo indígena mexicano.

De tal manera, los objetivos de investigación que se llevan a cabo son los siguientes: General. Describir, comprender y analizar las representaciones asociadas al proceso de envejecimiento rarámuri y el vínculo con modelos, saberes y formas de atención del continuo salud-enfermedad desde un enfoque intercultural, que considere las narrativas, actitudes, prácticas y comportamientos entre géneros y generaciones.

Específicos. a) Describir y determinar el contexto geográfico y sociocultural que ha permitido la persistencia de este grupo étnico y los dispositivos que han favorecido y/o afectado su calidad de vida en el envejecimiento, con énfasis en el proceso de salud/enfermedad/atención en la región, y b) Registrar y analizar las narrativas, las actitudes, las prácticas y los comportamientos de mujeres y varones rarámuri y externos al grupo, de diferentes generaciones, sobre las representaciones, continuidades, rupturas y/o reinvenciones del ser viejo(a) entre esta comunidad. 
La hipótesis central de la que se parte es: Si bien el envejecimiento es un proceso generalizado que implica una declinación funcional en sí mismo existen múltiples variantes que impactan a la persona rarámuri y su grupo de adscripción en este periodo del ciclo de vida, particularidades que no se observan en el desarrollo e implementación de políticas en el ámbito social, económico y de salud, lo que sin duda acentúa las desventajas acumuladas de este grupo étnico y arraiga las desigualdades estructurales que tanto los vulnera y excluye. Incluso, la edad que "acredita" ubicarse en el grupo de la tercera edad y que define la "ancianidad", en un nivel macroestructural, es de 65 años, lo que indudablemente no corresponde a la realidad rarámuri pues pese a la extrema pobreza, el alto grado de marginación y demás inconvenientes en los que perviven y resisten reducen la esperanza de vida y por ende la calidad de la misma, lo que se corroborará con la epidemiología clásica y sociocultural.

No obstante, la introyección de esta violencia simbólica es tal que desde la perspectiva rarámuri el bienestar y la calidad de vida es una constante que integra las vivencias cotidianas saludables y/o con presencia de enfermedad, es decir, el día a día se practica y re-significa más allá de la supuesta vulnerabilidad etaria material o simbólica al interior de su comunidad, sin embargo, al situarse ante la mirada de los mestizos y de la presencia de la sociedad nacional consideran manifiesta esa fragilidad fáctica, resultado de dichas desigualdades estructurales, mismas que se traducen en maltrato, incluso por las instituciones del sector público de salud, generalmente, por desconocimiento de las prácticas y significados culturales de este grupo étnico.

\section{Marco referencial}

Este apartado se presentará en dos grandes rubros. El primero desarrollará el marco conceptual que será la base de comprensión y análisis del tema seleccionado como son la vejez, el envejecimiento y el desarrollo adulto; la interculturalidad en salud, la exclusión social, el curso de vida y las desventajas acumuladas. El segundo se aproximará hacia el envejecimiento en población indígena y los cuerpos envejecidos en corporalidades rarámuri asociados a la salud y enfermedad de este grupo étnico.

\section{Envejecimiento y desarrollo adulto}

La vejez y el envejecimiento son fenómenos que deben estudiarse desde una perspectiva interdisciplinaria, debido a sus definiciones y relatos asociados diferenciados, interacciones múltiples y convergencias diversas entre un mosaico de campos teóricos y conceptuales que van desde la estructura globalizada que permite la reflexión sobre las políticas de seguridad institucional, económica y laboral; el vínculo entre éstas y los espacios local, regional y global, así como la desigualdad acumulada en el envejecimiento; los procesos de salud/ enfermedad/atención asociados al bienestar y calidad de vida; la promoción de un estilo de vida activo y saludable, y la implementación de espacios para ello; cruzando por la industria del cuidado del cuerpo con la juventud como valor, procesos de cuidado para enfermedades de duración considerable; la institucionalización de centros para la vejez; las redes solidarias intergeneracionales; la idea de corresponsabilidad asociada a la familia y no al Estado como institución; hasta las dinámicas de interrelación y transición entre la biografía y la historia en términos de configuración familiar, comunidad y cambio social; y las narrativas en términos de vivencias significativas y subjetivas pero a su vez polifónicas y polivalentes, entre otras (Montes de Oca, 2014, 2013).

Consideraremos a la adultez como la etapa cronológicamente más larga y a medida que avanza, las diferencias interindividuales se hacen más evidentes, debido a la importancia que cobran los factores sociales y culturales como condicionantes del desarrollo del ciclo de vida. Siguiendo a Papalia et al. (2009), el desarrollo adulto se considera como un proceso sistemático de cambio adaptativo en el comportamiento, debido a que permite lidiar 
con las condiciones de existencia, internas y externas, y las rutas a tomar pueden ser múltiples. Esto se debe tanto al aprendizaje, resultado de la experiencia, como a la maduración del cerebro y otros sistemas y estructuras físicas del organismo.

Así, el desarrollo puede considerarse como producto de múltiples fuerzas concurrentes que actúan sobre un sistema complejo. Al respecto Baltes (1987) señala al menos cinco características fundamentales en el desarrollo del ciclo de vida, a saber, dirección, dimensión y causalidad múltiple, plasticidad, e historia y contexto. De tal manera, una persona puede ubicarse en diversas edades de acuerdo con factores cronológicos, funcionales, biológicos, psicológicos y sociales.

\section{Interculturalidad en salud y exclusión social}

El proceso de interculturalidad ha estado presente en todo vínculo humano y ha sido registrado y analizado por las primeras teorías antropológicas. Recientemente Menéndez (2016a) en una reflexión crítica integra lo referente a las autonomías culturales y políticas de grupos étnicos, en donde se enfatiza la situación extrema de pobreza y salud de los pueblos indígenas resultado de la política de ajuste estructural del proceso migratorio masivo de la periferia al centro. Ahonda sobre la autonomía para una interculturalidad simétrica, es decir, considerar que cada grupo recodifica y resignifica los problemas e interpretaciones que se juegan en sus interrelaciones a partir de su propia matriz cultural.

Misma que supone reconocer que las relaciones culturales han coexistido de manera simultánea en contextos de dominación, explotación, hegemonía y subalternidad a la par de solidaridad, cooperación y adhesión. Siendo esta perspectiva la que orientará la lógica de dicha investigación.

La salud intercultural (Campos-Navarro, 2016) dejará de lado el proceso de despersonalización y cosificación de un padecimiento o enfermedad, considerando a la persona-paciente como una unidad somato-psíquica y socio-cultural. En donde el estudio y la atención de los pueblos originarios implique la comprensión y valoración de cada cultura en su ámbito local tanto en su lengua tradicional y alimentación local-regional como en su epidemiología sociocultural (saberes y prácticas de la medicina tradicional o de filiación cultural) y actividad que se realice en su cotidianidad y con base en su contexto geográfico. Además de considerar la coexistencia de los diversos sistemas de atención a la salud como parte intrínseca en toda sociedad, en donde el agente indígena de manera individual y colectiva tiende a integrar los saberes más que antagonizarlos, excluirlos o negarlos, unos en función de otros; pues los sintetizan, articulan, combinan, reconfiguran y organizan con base en sus condiciones étnico-culturales, político-económicas, eficacia técnica o de significación cultural, religiosas, entre otras.

El modelo médico será considerado como una construcción que supone en su estructura la producción teórica, técnica, ideológica y socioeconómica de los curadores, donde se incluyen los médicos; siendo a partir del proceso capitalista que se desarrollaron varios modelos de atención médica de tipo hegemónico, alternativo y el vinculado con la auto-atención (Menéndez, 2016b). ${ }^{3}$

No omito señalar, con base en la perspectiva que plantean Thomson (1993) y Bourdieu (1991) que este trabajo considerará como uno de los elementos guía al fenómeno y proceso de envejecimiento, es decir, a la vejez como experiencia humana individual y el contexto étnico, social,

3 En relación con los saberes y formas que se asocian al modelo de auto-atención, las primeras corresponden a las representaciones y prácticas organizadas como un entendimiento que opera a través de curadores, sujetos o colectivos; en tanto la segunda como experiencias utilizadas por los sujetos y grupos cuyo interés está en obtener trayectorias individuales (Menéndez, 2016c). Así, los saberes y formas brindan elementos para diagnosticar, explicar, atender, controlar, aliviar, aguantar, curar, solucionar o prevenir procesos que afecten la salud en términos reales o imaginarios, sin la intervención directa o intencional de curadores profesionales del campo de la biomedicina. 
económico y político en el que esta se produce, además de la cualidad en que las sociedades envejecen en el contexto global. Esto es, se vinculará la experiencia intercultural de los rarámuri en el contexto local y global, con su consecuente resignificación y reapropiación en el ámbito de su salud y bienestar.

En primera instancia cabe mencionar que los fenómenos culturales son constructos significativos que están insertos en relaciones de poder y de conflicto y que son producidos o actuados siempre en circunstancias sociohistóricas que poseen distintos niveles de poder y autoridad (Thompson, 1993). Así la cultura se encuentra mediada por los agentes sociales, que se encuentran situados en contextos socialmente estructurados, $y$, por lo tanto, jerarquizados y vinculados a relaciones de distintas fuerzas.

Las formas simbólicas tienen una carga valorativa y normativa que se expresa tanto en los elementos de cultura objetivada (manifiesta en relaciones de parentesco, rituales, formas de organización social, división sexual del trabajo, entre otras) como en la cultura internalizada o subjetivada, entendida como visión del mundo o esquemas de percepción, interpretación o acción, que existen bajo la forma de habitus. ${ }^{4}$

De igual manera, la perspectiva económica-política enfatiza las relaciones sociales que envuelven la asignación y control de las actividades en diversos campos de poder, en donde los individuos tratan de generar un balance entre la posibilidad de conflicto y el conocimiento con base en la experiencia personal, el conocimiento cultural

$4 \quad$ Bourdieu define al habitus como el sistemas de disposiciones duraderas y transferibles, estructuras estructuradas predispuestas para funcionar como estructuras estructurantes, es decir, como principios generadores y organizadores de prácticas y representaciones que pueden estar objetivamente adaptadas a su fin sin suponer la búsqueda consciente de fines y el dominio expreso de las operaciones necesarias para alcanzarlos, objetivamente $<<$ reguladas $>>$ $\mathrm{y}<<$ regulares $>>$ sin ser el producto de la obediencia a reglas, $y$, a la vez que todo esto, colectivamente orquestadas sin ser el producto de la acción organizadora de un director de orquesta (Bourdieu, 1991, p. 92). local y los recursos disponibles para satisfacer sus objetivos específicos. De tal manera que las respuestas y sus consecuencias difieren dependiendo de sus contextos y posicionamientos individuales con sus redes sociales, es decir, comportamientos "lógicos" y "apropiados" pueden diferir con base en la clase, el género, la etnicidad y la generación, principalmente. Este enfoque es útil para la antropología debido a que toma en cuenta las contradicciones y complejidades de la vida social y cómo estas influencian a la biología, pues enfatiza los problemas en donde las personas deben confrontar y hacer frente a su propia capacidad, condicionados por los recursos y materiales sociales disponibles.

En este orden de ideas, el concepto exclusión social asociado a los poderes económicos y políticos de las sociedades a lo largo del tiempo, ha derivado y se ha vinculado con términos tales como pobreza, marginación y desigualdad identificadas con la noción de desarrollo e indicadores de carácter cuantitativo principalmente; así como inserción, integración social (cultural, normativa, comunicativa y funcional) y ciudadanía asociadas con la supresión del goce de derechos y obligaciones en modelos de expresión globalizada y neoliberal. ${ }^{5}$

Por lo que, la exclusión será entendida como un fenómeno estructural. Un proceso dinámico, multidimensional y politizable que potencia los riesgos de vulnerabilizar a ciertos colectivos, tiene causas y consecuencias políticas, económicas, culturales y temporales, pues compromete la supervivencia de las generaciones futuras y prepara las exclusiones del mañana (Bessis, 1995), no obstante,

\footnotetext{
5 La génesis del término fue por el padre Joseph Wresinski en el Movimiento de ayuda a toda desgracia - Cuarto mundo, originado en la Asociación contra la exclusión de los más pobres en Noisy-Le Grand, Francia, en 1957; y desarrollado por el editor René Lenoir en 1974 quien señalaba la fractura de los lazos sociales y a aquellas personas que estaban siendo apartadas del mercado laboral y de los beneficios sociales, por lo que dicho concepto no podía separarse del entorno de los Estados-Nación. No obstante, la idea fue abordada des-

de las ciencias sociales por Durkheim, Mertón y Marx quienes hacían cierta referencia a estos criterios en sus obras con los términos anomia y alienación, respectivamente; aunque en la actualidad se han ido perfilado con matices más precisos al modelo hegemónico mundial (Rizo, 2006).
} 
podría y debería interrumpirse desde las políticas públicas, las prácticas institucionales y la propia acción colectiva (Rizo, 2006). En palabras de Estivil (2003, pp. 20-30) la exclusión social implica una "acumulación de procesos confluyentes con rupturas sucesivas que, arrancando del corazón de la economía, la política y la sociedad, van alejando e 'inferiorizando' a personas, grupos comunidades y territorios con respecto a los centros de poder, los recursos y los valores dominantes".

Siguiendo a Rizo (2016) destacamos las manifestaciones y formas de exclusión como múltiples y con la posibilidad de reconocerlas en función de la fase en que se encuentren las personas implicadas, a saber, inicio, recuperación, deterioro o cronificación; del tipo de dominancia en los factores materiales o sociales; del papel que juegan las redes sociales primarias; principalmente, mismas que pueden aparecer en el campo territorial, por motivos religiosos, étnicos, biológicos, ideológicos, de género, del conocimiento técnico, de la educación, de la asistencia sanitaria, entre otros; lo que será base en el desarrollo de la investigación.

\section{Curso de vida, desventajas acumuladas}

Ante este panorama el curso de vida, como una orientación teórica y metodológica, cruza disciplinas, categorías del conocimiento y fronteras culturales para ofrecer un marco descriptivo y explicativo, en este caso en el proceso de envejecimiento rarámuri. Nos permitirá cavar profundo sobre la complejidad de la vida de este grupo étnico que habita Munérachi, en donde se entrelazan fuerzas interpersonales, estructurales e históricas (Elder Jr., Kirkpatrick y Crosnoe, 2003). Los ejes dinámicos de esta categoría, curso de vida, reflejan la naturaleza temporal de las vidas, es decir, capturan el movimiento a lo largo de los tiempos biográficos e históricos y son: trayectoria sobre la línea o carrera de vida, la cual puede cambiar de dirección, grado y proporción; transición que marca los cambios de estado, posición o situación según el timing, secuencia y duración; y punto de inflexión o turning point, retrospectivo, que también aborda los cambios de estado desde la discontinuidad en una o más trayectorias vitales.

La teoría de las desigualdades acumuladas asociada al curso de vida (Ferraro y Shippee, 2009) integra elementos micro, meso y macro sociológicos. Reconoce la multidisciplinariedad y busca vínculos con modelos biológicos asociados con la edad, así como fuerzas sociales cotidianas que pueden abarcar cambios psicológicos y biológicos en el cuerpo; pone atención en el papel de la reproducción, el parentesco o linaje familiar, los genes, el contexto ambiental y el estatus o jerarquía social. Refiere que la inequidad conduce a la acumulación de materiales biológicos debajo de la piel, que son marcadores del envejecimiento y predictivo de la senescencia. También plantea que el sistema social genera inequidad tanto en los procesos de desarrollo y demográficos como en las trayectorias personales, a través de la acumulación del riesgo, los recursos disponibles, las trayectorias percibidas y la agencia humana. No obstante, plantea que los periodos críticos no necesariamente son inexorables, pues muchas de esas impresiones tempranas en el organismo pueden ser neutralizadas o cambiadas de sentido; incluso los recursos con los que se cuenten pueden moderar los efectos adversos. Por lo tanto, la teoría de la desigualdad acumulada es un proceso de curso de vida y no solo la diferencia que viene tempranamente con la adversidad en dicha inequidad.

\section{Envejecimiento en población indígena}

Los grupos indígenas representan el $5 \%$ de la población mundial, aproximadamente el $15 \%$ se encuentran en pobreza y el resto en extrema pobreza, habitando áreas rurales (INEGI, 2010). En México el 7\% de la población es indígena, se caracteriza por niveles altos de marginación y brechas e inequidad social existentes (Leyva Flores, Servan Mori, Infante Xibille, Pelcastre Villafuerte y González, 2014). Alrededor del $44 \%$ reside en municipios y viven en extrema pobreza, de acuerdo con los indicadores del Con- 
sejo Nacional de Evaluación de la Política de Desarrollo Social (CONEVAL, 2013) esta situación marginal se debe mayoritariamente al ingreso económico y pese a que este se destina exclusivamente a la compra de alimento, los nutrientes ingeridos son insuficientes para tener una vida saludable, ende están por debajo del nivel de bienestar esperado y son vulnerados en su salud y en las demás esferas del ámbito de lo social (Servan Mori, Pelcastre Villafuerte, Heredia Pi y Montoya Rodríguez, 2014).

El envejecimiento de la población en México se hace evidente al comparar los porcentajes de población en las cohortes de edad, en 2015 la población menor de 15 años representó $27.4 \%$ del total, la que se encuentra entre los 15 y 64 años, 65.4\%, y la población en edad avanzada o de 65 años y más, 7.2\% (CDI, 2016). La población indígena no ha estado al margen del proceso de crecimiento de la población en edad avanzada. La Comisión Nacional para el Desarrollo de los Pueblos Indígenas de México (CDI, 2016) con base en los resultados de la Encuesta Intercensal 2015, contabilizó alrededor de 12 millones de indígenas de los cuales, la población de 60 años o más representó el $10.4 \%$, que significa un incremento de casi $3 \%$, con respecto al valor registrado en el año 2000 , de $7.3 \%$. De estos, los adultos mayores indígenas hablantes de lenguas originarias suman más de $1 \mathrm{mi}-$ Ilón de personas. Cuatro de cada cinco adultos mayores hablantes de lengua son monolingües y solo $18 \%$ habla además el español.

En el estado de Chihuahua los adultos mayores representan el $8.3 \%$ (282.725) de la población total, de ésta el $49.6 \%$ son varones y el $50.3 \%$ mujeres. En Batopilas, el municipio que nos ocupa, la distribución de personas de más de 60 es de $8.0 \%$. La esperanza de vida al nacimiento en el estado para 2010 era de 66 y 75 años para varones y mujeres, respectivamente. Los adultos mayores indígenas respecto a la población total de adultos mayores del Estado son del 3.4\% $(9,768)$ (INAPAM, 2015). Munérachi una de las localidades de Batopilas, habitada comple- tamente por rarámuris, en 2010 contaba con 113 indígenas: 57 varones y 56 mujeres (SEDESOL, 2013). Cabe señalar que la unidad de microrregiones del SEDESOL no distingue entre sexo y generación. Además de que la encuesta intercensal del INEGI 2015 sólo maneja los datos de localidades de menos de 50 mil habitantes.

\section{El contexto rarámuri}

Los rarámuri o pies ligeros habitan la parte de la Sierra Madre Occidental que atraviesa el Estado de Chihuahua y el suroeste de Durango y Sonora. La topografía de la región tiene elevaciones superiores a los $2.000 \mathrm{msnm}$ y depresiones en la cima de los barrancos de $600 \mathrm{msnm}$. Esta área se dividió en tres grandes regiones demarcaciones o sub-regiones: cumbres o alta tarahumara, barrancas o baja tarahumara y en la región oriental o de las praderas (Pennington, 1963), mismas que han estado interrelacionadas entre sí por las particularidades ecológico-sociales que las constituyen.

La diversidad intercultural se manifiesta al compartir esta región con otros grupos étnicos como los ódame-tepehuanes, o'oba-pimas bajos, warijó-guarijíos y mestizos. Los rarámuri se concentran en 17 municipios, siendo Batopilas y Guachochi algunos de los que destacan por su alta densidad de población indígena. Se han registrado 70,842 hablantes de tarahumara en el estado de Chihuahua y 75,545 a nivel nacional; cabe señalar que la lengua rarámuri forma parte del tronco yuto-nahua, de la familia taracahita (CDI, 2017).

El grupo étnico que nos ocupa ha sido objeto de un gran número de expediciones e investigaciones de carácter antropológico desde 1900 por autores extranjeros y nacionales, ${ }^{6}$ sobre su historia, organización política y de subsistencia, lengua, cosmovisión, entre otras; no así para abordar el proceso de envejecimiento asociado a la salud. Algunas de ellas han sido financiadas por instituculturas.
Por cronistas y misioneros desde 1607, en el encuentro de las dos 
ciones académicas y gubernamentales. En el caso mexicano los resultados obtenidos fueron instrumentados en políticas de Estado para el desarrollo y la integración indigenista en la Sierra Tarahumara.

Los trabajos clásicos que destacan algunos referentes sobre la salud rarámuri son los de Carl Lumholtz en 1902 (1986), Wendel Bennet con Robert Zingg en 1935 (1986) y Fructuoso Irigoyen desde 1974 (2015) sobre prácticas de medicina tradicional; Carlos Basauri (1929), la constitución antropológica del cuerpo; Robert Bye (1986), estudio etnobotánico comparativo en plantas medicinales; William Merrill en 1988 (1992), cosmovisión del cuerpo; principalmente. Recientemente Francisco Cardenal (1993) ahonda sobre la medicina tradicional local como un sistema médico con simbolizaciones y exégesis de la enfermedad y su causalidad; Olga Sánchez (1996) realizó una tipología de las enfermedades tradicionales en Narárachi; Silvia Ortíz (1999) integró una coordinación de la medicina en el norte de México; Eugeni Porras (2002) desde la política indigenista en la Sierra Tarahumara se aproxima al proceso de salud a la llegada de los servicios de salud; Juan Luis Sariego (2002) aborda las repercusiones de la política indigenista en la región $y$, aunque no es su eje, de manera tangencial los cambios y resistencias culturales vinculados al sistema médico hegemónico.

La estructura social rarámuri ${ }^{7}$ se ha desarrollado a lo largo del proceso de su formación étnica, ha incorporado de forma importante elementos del pasado precolombino, en el sentido de las prácticas de apropiación y de transformación medio ambiental de aquellos tiempos. Basadas éstas en dinámicas de recolección y caza, así como de una rudimentaria agricultura en donde mujeres y varones participaban, lo que implicaba una frecuente movilidad espacial, organizada en pequeñas bandas o

$7 \quad$ Siguiendo a Radcliffe Brown (1974), considero la estructura social como un referente empírico de la red de relaciones en que se conectan las diferentes comunidades humanas. núcleos sociales que generaban capacidades productivas y de desarrollo similares para todos sus miembros, desarrollando así un formato de individualidades autonómicas que participarían a través de la cooperación y reciprocidad en la reproducción de la comunidad. A su vez estas bandas se relacionaban con otras cercanas al ámbito territorial conformando núcleos humanos más extensos, en función de reuniones festivas mediadas por la cerveza de maíz (Noriega, 1992).

Es decir, se trata de comunidades étnicas fundadas en un modelo agrícola-campesino que interactúan de manera cotidiana con prácticas recolectoras bajo un calendario festivo-ritual que implica la forma de relacionarse con el grupo, con la tierra y con el cosmos en cada fase del ciclo agrícola: siembra, cultivo y cosecha. Ello se aprende mediante la tradición oral que forma una "memoria permanente del sentido individual y social de estar en el mundo. Por ello en la cultura rarámuri se hacen discursos, se organizan fiestas y se elabora cerveza de maíz con el fin de embriagarse, bailar y asegurar el orden del cosmos" (Gotés, 2012, p. 35).

Los pueblos rarámuri hoy recuperados en su mayoría como propiedades ejidales se constituyen por un conjunto de rancherías, y una ranchería de 2 a 12 casas en promedio distribuidas en una extensión no menor a 50 has., que tienen como eje un centro ceremonial. Una familia rarámuri regularmente tiene de una a dos casas junto a la parcela, en donde pasan ciertas temporadas en cada una de ellas para barbechar, sembrar, cultivar y obtener la cosecha, además de socializar con las demás familias de su ranchería.

Munérachi es una ranchería o localidad habitada totalmente por población indígena, no cuenta con servicios como agua entubada, drenaje, ni energía eléctrica; las viviendas son de un solo cuarto y con piso de tierra. La única infraestructura pública con la que se cuenta es la escuela albergue. Recientemente, desde hace un par de 
años atrás, se ha iniciado con el proceso de electrificación, no obstante, el avance es relativamente lento debido a las condiciones de acceso.

\section{El alma rarámuri: la salud y la enfermedad}

... a través de un soplido Onorúami hizo lo que hay sobre la tierra. ${ }^{8}$

La percepción del mundo rarámuri es animista, todo ser vivo está dotado de un alma, iwigála, espacio en donde se materializa el bien y el mal y, por ende, la salud y la enfermedad. Para poder comprender su medicina habrá que acercarse a la estructura de su cosmovisión y al lugar que ocupan en el universo.

El concepto de alma rarámuri "define la esencia de la existencia humana en los planos biológico, espiritual y social (y la manera en que) se relacionan con los mundos moral y natural. Participa directamente al motivar y justificar acciones prácticas (entre ellas) la salud y la muerte" (Merrill, 1992, p. 273).

Montemayor (1999, pp. 45-50) considera que los rarámuri reconocen en lo celeste y en lo terrenal "derroteros precisos por los que deben atravesar; caminando bien, para el sostenimiento del mundo". Plantea que en este grupo étnico existe la consciencia de una responsabilidad con la tierra, gawi, con su mundo; tanto en las actividades agrícolas que han Ilevado a cabo desde tiempo precolombinos como en su pensamiento, conducta y orden ritual, lo que se refleja en lo cotidiano y en lo festivo mediante sus ceremonias, ritos y danzas, lo que en conjunto ayuda al equilibrio de la vida. "La primera faz de este compromiso es el conocimiento (mismo de dicha) responsabilidad y (después su) transmisión: es necesario recordarlo como conocimiento (que pasa) de los abuelos (...) a los nietos para que no se pierda. Los siguientes

8 Mito: Origen de la vida (Mondragón, Tello y Valdéz, 1995, p. 27). rasgos (...) son la fortaleza moral ante el sufrimiento y el apoyo a los (otros) rarámuri".

Además de estos referentes en la socialización de los infantes, los discursos ceremoniales o sermones, nawésari, pronunciados por la autoridad tradicional indígena, el gobernador, también transmiten de manera clara y contundente dicho ideal; enfatizan la misión de sostener el mundo. Al respecto Merrill (1992, p.104) estipula con base en la raíz na-pensar de las palabras nawésari y nátiri, que las personas que actúan apropiadamente son caracterizadas no solo como 'gente buena' sino como 'buenos pensadores'.

Este planteamiento se refuerza a través del relato etnohistórico de González Rodríguez (1987, p. 416), recupera extractos de algunos sermones, a saber, omarúame tibupo keta gawiwa, tengamos cuidado de todo nuestro mundo, 'cuyos pilares en verdad somos nosotros'; pe tamujé rewegachi tónara kam-pa, 'nosotros somos las columnas del cielo'. De igual manera señala que el tarahumar gentil o cimarrón, conocido así desde la época colonial, "cree que si ellos se bautizan, ellos como las columnas se resquebrajarán y el firmamento se les vendrá encima apachurrándolos a todos. Sería el desplome del sol, la luna, los astros, y la destrucción del mundo".

Mira hijo -le dijo un viejito a su hijo-. Tú serás el que fortalezca el mundo. Tú transmitirás todo lo que te he dicho siempre. Si no lo dices a otros, se perderá. "Nosotros somos los rarámuri. Nosotros somos el pilar del mundo. Tenemos que recordar lo que decían los antepasados, así seremos más rarámuri. No hay que entristecernos si nos hacen sufrir. Hay que ser fuertes (...) Si recoges mucho maíz, compártelo cuando alguien te visite (...). (Testimonio de Baptista, (Trad.) Montemayor, 1999, p. 4 6)

De esta manera, a partir de las crónicas registradas de por lo menos la segunda mitad del siglo XVII hasta las narrativas etnográficas contemporáneas sobre la población 
rarámuri podemos observar la persistencia, resistencia y resignificación de su cosmovisión en el devenir histórico. Finalmente, resta señalar que la mayoría de las enfermedades presentan un vínculo con el alma, ya que esta constituye la sustancia principal de la vida. Por ejemplo, las enfermedades infecciosas, heridas corporales o cualquier patología considerada de orden biológico pueden poner en peligro la vida rarámuri, no propiamente por las características intrínsecas de la misma, sino porque al afectar el cuerpo del paciente provocan que el alma no se sienta cómoda y eventualmente llegue a abandonarlo y causar así la muerte. También las enfermedades que son por ataque directo del alma se consideran más graves debido a que esta pudo ser capturada por un hechicero mientras la persona dormía; el alma se sintió incomoda porque se bebió demasiado y se salió del cuerpo; o porque algún ser de los aguajes o plantas la secuestró por alguna ofensa cometida. De tal manera que, si el alma no vuelve al cuerpo, el paciente muere (Merrill, 1992; Acosta, 2011).

Al respecto Cardenal (1993, pp. 27-28) señala que el concepto de enfermedad es anímico y no orgánico, iniciando a partir de la tristeza como primer síntoma pues considera a los rarámuri como una sociedad de la alegría, pues esta emoción es fundamental para su cosmovisión y día a día. Con el siguiente testimonio se refiere que la tristeza y el dolor se pueden ver y así enfermar a otras personas sanas, a saber: “... no, no voy a ir al tesgüino porque ando enferma, me duele esta cabeza y ando poco triste (...) No quiero enfermar a los otros." (Testimonio Rosaria, San José Cuechi, Batopilas, 2016).

\section{Referencias}

Acosta, L. (2011). Owirúame y doctor: Conflictos, negociaciones e intercambios de dos mundos. Tesis de Licenciatura en Antropología. México: ENAH - Unidad Chihuahua.

Baltes, P. B. (1987). Theoretical propositions of lifespan development psychology: On the dynamics between grown and decline. Developmental Pcychology, 23(5), pp. 611-626.
Basauri, C. (1929). Monografía de los tarahumaras. México: Talleres gráficos de la Nación.

Bennet, W. y R. Zing. (1986). [1935]. Los tarahumaras. Una tribu india del norte de México. Clásicos de la Antropología. México: Instituto Nacional Indigenista.

Bessis, S. (1995). De la exclusión social a la cohesión social, Síntesis del Coloquio de Rosklide. (2-4 marzo). Universidad de Rosklide.

Bourdieu, P. (1991). El sentido práctico. Madrid: Taurus.

Bye, R. (1986). Medicinal plants of the sierra madre: Comparative study of tarahumara and Mexican market Botany. January. 40(Issue 1), pp. 103-124.

Campos-Navarro, R. (2016). "Enseñanza de la antropología médica y la salud interculturalidad en México". En Campos-Navarro, R. (Comp.) Antropología médica e intreculturalidad, México: UNAM, McGraw-Hill/Interamericana Editores.

Cardenal, F. (1993). Remedios y prácticas curativas en la Sierra Tarahumara. México: Gladius.

CDI - Comisión Nacional para el Desarrollo de los Pueblos Indígenas. (2016). Indicadores sobre adultos mayores indígenas de México. [En línea] <<https://www.gob.mx/cdi/articulos/indicadores-sobre-adultos-mayores-indigenas-de-mexico >>

(2017). [En línea] <<http://www.cdi.gob.mx/pueblos_mexico/tarahumaras.pdf $>>$

CONEVAL - Consejo Nacional de Evaluación de la Política de Desarrollo Social. (2013). Informe de evaluación específica de desempeño 2012-2013: Seguro Popular. [En línea] <<http://www.coneval. gob.mx/Evaluacion/Eval_Especificas/Paginas/EED_2012-2013/ EED_2012_2013_SALUD.aspx>>

Duch, L. (2002). Antropología de la vida cotidiana. Simbolismo y salud. Madrid: Trotta.

Elder Jr., Glen H., Kirkpatrick J. y Crosnoe, R. (2003). “The Emergence and Development of Life Course Theory". En Mortimer, J. y Shanahan, M. (Eds.). Handbook of the Life Course, New York: Kluwer Academic/ Plenum Publishers.

Estivill, J. (2003). Panorama de la lucha contra la exclusión social. Conceptos y estrategias. Ginebra: Oficina Internacional del Trabajo.

Ferraro, K y Shippee, T. P. (2009). Aging and Cumulative Inequality: How Does Inequality Get Under the Skin? The Gerontologist, .9(3), pp. 333-343.

Geertz, C. (2006). “Descripción densa: hacia una teoría interpretativa de la cultura". En La interpretación de las culturas. España: Gedisa. González Rodríguez, L. (1987). Crónicas de la Sierra Tarahumaras. Col. Cien México. SEP. 
Gotés Martínez, L. (2012). Los pueblos indígenas de Chihuahua. México: INAH.

INAPAM - Instituto Nacional de las Personas Adultas Mayores. (2015). Perfil Demográfico, Epidemiológico y Social de la Población Adulta Mayor en el País. Una Propuesta de Política Pública: SEDESOL, Dirección de Atención Geriátrica.

INEGI - Instituto Nacional de Estadística Geografía e Informática. (2010). Principales resultados del Censo de Población y Vivienda del 2010. [En línea] http://www.inegi.org.mx

org.mx>>

(2015). Encuesta intercensal. [En línea] <<http://www.inegi.

Irigoyen-Rascon, F. (2015) [1974]. Tarahumar medicine, ethnobotany and healing among the raramuri. EE.UU.: University of Oklahoma Press.

Leyton, C. y Muñoz, G. (2016). Revisitando el concepto de exclusión social: su relevancia para las políticas contra la pobreza en América Latina. Revista del CLAD Reforma y Democracia.(6), pp. 39-68.

Leyva-Flores, R., Servan-Mori, E., Infante-Xibille, C., Pelcastre-Villafuerte, B.E. y González, T. (2014). Primary Health Care Utilization by the Mexican Indigenous Population: The Role of the Seguro Popular in Socially Inequitable Contexts. PLoS ONE 9(8), e102781. doi:10.1371/ journal.pone. 0102781

Lumholtz, C. (1986). [1902]. El México desconocido, Tomo I. Edición facsimilar. México: Instituto Nacional Indigenista.

Menéndez, E. (2016a). Cuestiones metodológicas sobre antropología e interculturalidad. En Campos-Navarro, R. (Comp.) Antropología médica e interculturalidad, México, UNAM: McGraw-Hill/Interamericana Editores.

(2016b). “Modelos hegemónico, subalterno y de autoatención". En Campos-Navarro, R. (Comp.) Antropología médica e interculturalidad. México: UNAM: McGraw-Hill/Interamericana Editores.

(2016c). Modelos, saberes y formas de atención de los padecimientos. En Campos-Navarro, R. (Comp.) Antropología médica e interculturalidad. México: UNAM: McGraw-Hill/Interamericana Editores.

Merrill, W. (1992). [1988]. Almas rarámuris. México: Instituto Nacional Indigenista.

Montemayor, C. (1999). [1995]. Los tarahumaras. Pueblo de estrellas y barrancas. México: Editorial Aldus.

Montes de Oca Zavala, V. (Coord.). (2013). Envejecimiento en América Latina y el Caribe: enfoques en investigación y docencia de la Red Latinoamericana de Investigación en Envejecimiento (LARNA). México: Instituto de Investigaciones Sociales, UNAM. nes

(2014). Vejez, salud y sociedad en México: aproximaciodisciplinarias desde perspectivas cuantitativas y cualitativas. México: UNAM.

Noriega, F. (1992). De primitivos a campesinos. Tarahumares. Evolución y cambio cultural 1600/1890. Tesis para optar por el grado de maestro en Antropología. México: El Colegio de Michoacán.

OMS - Organización Mundial de la Salud. (2015). Informe Organización Mundial de la Salud. [En línea] <<http://www.who.int>>

ONU - Organización de Naciones Unidas (2015). Agenda 2030. [En línea] $\quad<<$ https://sustainabledevelopment.un.org/post2015/transformingourworld $>>$

Papalia, D., Sterns, H., Feldman, R., \& Camp, C. (2009). Desarrollo del adulto y vejez. México: McGraw-Hill.

Pennington, C. (1963). The tarahumar of Mexico Their environment and material culture, University of Utah Press: Salt Lake.

Porras, E. (2002). Políticas indigenistas en la Sierra Tarahumara. México. Instituto Chihuahuense de la Cultura.

Radcliffe-Brown, A.R. (1974). Estructura y función en la sociedad primitiva, Barcelona: Ediciones Península.

Rizo López, A. E. (2006). ¿A qué llamamos exclusión social? En Polis Revista Latinoamericana Persona y Otredad, 15|2006, Centro de investigación sociedad y políticas públicas (CISPO). [En línea] <<http:// polis.reveus.org/5007>>, pp. 1-17.

Sánchez, O. (1996). Sistema médico tradicional rarámuri: elementos de unificación cultural en la comunidad de Narárachi. México: ENAH.

Sariego-Rodríguez, J.L. (2002). El indigenismo en la Tarahumara: identidad, comunidad, relaciones interétnicas y desarrollo en la Sierra de Chihuahua. México: Instituto Nacional Indigenista / Consejo Nacional para la Cultura y las Artes / INAH.

SEDESOL - Secretaría de Desarrollo Social. (2013). [En línea] $<<$ http://www.microrregiones.gob.mx/catloc/contenido.aspx?refnac $=080080553>>$

Servan-Mori, E., Pelcastre-Villafuerte, B., Heredia-Pi, I. \& Montoya-Rodríguez, A. (2014). Essential Health Care Among Mexican Indigenous People in a Universal Coverage Context. Ethnicity \& Disease, (24), Autumn.

Thomson, J. (1993). El concepto de cultura. En Ideología y cultura moderna. Teoría crítica social en la era de la comunicación de masas. México: UAM. 